\title{
Pharmacokinetic and Absolute Bioavailability Study of Total Panax Notoginsenoside, a Typical Multiple Constituent Traditional Chinese Medicine (TCM) in Rats
}

\author{
Xiaoyu Li, ${ }^{a}$ Guangji WANG, ${ }^{*}, a$ Jianguo Sun, ${ }^{a}$ Haiping HaO, ${ }^{a}$ Yuqing XIOng, ${ }^{b}$ Bei YAN, ${ }^{a}$ \\ Yuanting ZHENG, ${ }^{a}$ and Longsheng SHENG $^{c}$ \\ ${ }^{a}$ Key Laboratory of Drug Metabolism and Pharmacokinetic, China Pharmaceutical University; Nanjing, 210009, China: \\ ${ }^{b}$ Institute of Clinical Pharmacology, Medical College of Nanchang University; Nanchang, 330006, China: and ${ }^{c}$ Center for \\ Instrumental Analysis, China Pharmaceutical University; Nanjing, 210009, China. \\ Received November 19, 2006; accepted February 2, 2007
}

LC/ESI/MS method was employed for the pharmacokinetic evaluation of total panax notoginsenoside (TPNS) in rats. After oral or intravenous administration of TPNS at the dosage of 300.0 or $10.0 \mathrm{mg} \mathrm{kg}^{-1}$ to rats respectively, panax notoginsenoside $R 1$, ginsenoside $R g 1, R d$, $R e$ and $R b 1$ were simultaneous determined in rat plasma. Pharmacokinetic parameters and absolute bioavailability of panax notoginsenoside R1, ginsenoside Rg1, Rd, Re and Rb1 were obtained by the Drug And Statistics for windows (DAS) pharmacokinetic software. The pharmacokinetic parameters of all analytes were different form each other. $T_{1 / 2}$ were changed from 0.72 to $22.16 \mathrm{~h}$ and $A U C$ were changed from 1.03 to $98.94 \mathrm{mg} / \mathrm{l} \cdot \mathrm{h}$ after oral or intravenous administration TPNS or Xuesaitong (TPNS) injection. The absolute bioavailability of R1, Rg1, Rd, Re and Rb1 were of 9.29\%, $6.06 \%, 2.36 \%$, $7.06 \%$ and $1.18 \%$, respectively.

Key words pharmacokinetic; absolute bioavailability; total panax notoginsenoside (TPNS); traditional Chinese medicine (TCM); assaying

San-Chi, the roots of panax notoginsenoside Radix (BURK) F. H. CHEN is a kind of herb belongs to Acanthopanax Gracilistylus with synonym of stephania sinica and pseudoginsen radix. It has been considered to be one of the famous traditional Chinese medicinal herbs in China or other oriental countries for thousands of years. ${ }^{1)}$ San-Chi contains many of chemical constituents. Total panax notoginsenoside (TPNS), isolated from San-Chi is a mixture of more than 20 Dammarane type saponins, including ginsenoside Rg1, Rg2, $\mathrm{Rb} 1, \mathrm{Rb} 2, \mathrm{Rb} 3, \mathrm{Rc}, \mathrm{Rd}, \mathrm{Re}, \mathrm{Rh}, \mathrm{F} 2$ and notoginsenoside R1, R2, R3, R4, R6, Fa, Fc, Fe, etc. Among these constituents, panax notoginsenoside $\mathrm{R} 1$, ginsenoside $\mathrm{Rg} 1, \mathrm{Rd}, \mathrm{Re}$ and $\mathrm{Rb} 1$ (Fig. 1) was considered to be the principal active constituents. ${ }^{2,3)}$ They are all derivatives from $20(S)$-protopanaxadiol, $20(S)$-protopanax-panaxatriol or Dammarane type triterpene. ${ }^{4)}$ TPNS displays important role on the treatment of hematinic, anti-inflammatory, coronary heart disease, the sequelae of cerebrovascular accident, anti-fatigue, hepato-

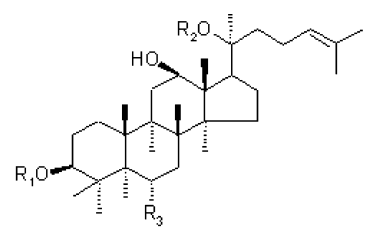

\begin{tabular}{cccc}
\multicolumn{4}{c}{$\mathrm{a}$} \\
\hline & $\mathrm{R}_{1}$ & $\mathrm{R}_{2}$ & $\mathrm{R}_{3}$ \\
\hline $\mathrm{Rl}$ & $\mathrm{H}$ & $-\mathrm{Glc}$ & -OGlc(2-1)Xyl \\
$\mathrm{Rgl}$ & $\mathrm{H}$ & $-\mathrm{Glc}$ & - OGlc \\
$\mathrm{Rd}$ & - Glc(2-1)Glc & - Glc & $\mathrm{H}$ \\
$\mathrm{Re}$ & $\mathrm{H}$ & $-\mathrm{Glc}$ & - OGlc(2-1)Rha \\
$\mathrm{Rb1}$ & - Glc $(2-1) \mathrm{Glc}$ & - Glc(6-1)Glc & $\mathrm{H}$ \\
\hline
\end{tabular}

Fig. 1. Chemical Structure of Panax Notoginsenoside R1, Ginsenoside Rg1, Rd, Re, Rb1 (Part a) and Internal Standard Digoxin (Part b) protection, anticancer and immunological disease ${ }^{5)}$ as well as ginsenoside. ${ }^{\text {) }}$ Many preparations of TPNS were used in clinic including tablet, drop pill and injection. Xuesaitong injection was one of these preparations which only consisted of TPNS. $^{7)}$

As a kind of typical multiple constituent and multiple actions traditional Chinese medicine (TCM), it was very difficult to evaluate the pharmacokinetic profiles of TPNS in rats or human. Only several literatures reported recent years for the pharmacokinetic study of TPNS (powder or liposomes) according to the evaluation of ginsenoside $\mathrm{Rg} 1$ and $\mathrm{Rb} 1$ after oral, intravenous and pulmonary instillation administration TPNS in rats. ${ }^{8-10)}$ It was not perfect for the pharmacokinetic evaluation of TPNS when panax notoginsenoside R1, a kind of typical constituent in San-Chi was missed.

The development of analytical technique gives us a possibility for the trace determination of constitutent of TCM in bio-sample. In this contribution, a developed and validated LC-ESI-MS method was employed for the simultaneous determination of panax notoginsenoside R1, ginsenoside $\mathrm{Rg} 1, \mathrm{Rd}, \mathrm{Re}$ and $\mathrm{Rb} 1$ in rat plasma. A reasonable and general pharmacokinetic profile of TPNS in rats was obtained according to the evaluation of absolute bioavailability and pharmacokinetic profiles of the consitutents mentioned above.

\section{MATERIALS AND METHODS}

Chemicals and Reagents Acetonitrile was of HPLC grade and purchased from Merck, Darmstand, Germany. Total panax notoginsenoside (TPNS), Panax notoginsenoside $\mathrm{R} 1$, ginsenoside $\mathrm{Rb} 1, \mathrm{Rd}$ were kindly provided by Yunnan Plant Pharmacy Co., Ltd. Ginsenoside Rg1, Re and digoxin (IS) (Fig. 1) were purchased from Chinese National Institute for the Control of Pharmaceutical and Biological Products, 
Beijing, China. Xuesaitong injection (consisting of $50 \mathrm{mg} / \mathrm{ml}$ TPNS) was kindly provided by Jiamusi Xinchen pharmaceutical Co., Ltd., Heilongjiang, China.

Liquid Chromatography Conditions Mobile phase: A, $0.001 \%$ formic acid; B, acetonitrile. Gradient elute procedure: $0-37 \mathrm{~min}, \mathrm{~B} 18.5 \%$; $37-45 \mathrm{~min}$, B $18.5-35 \%$; $45-$ $50 \mathrm{~min}, \mathrm{~B} 35-45 \%$; 50.03-60 min, B $18.5 \%$. Column: Hypersil ODS, $250 \times 5.0 \mathrm{~mm}$, maintained at $40{ }^{\circ} \mathrm{C}$ with flow rate of $1.0 \mathrm{ml} / \mathrm{min}$. Assaying of panax notoginsenoside R1, ginsenoside $\mathrm{Rg} 1, \mathrm{Rd}, \mathrm{Re}$ and $\mathrm{Rb} 1$ in TPNS powder and Xuesaitong (TPNS) injection was performed under this condition.

Mobile phase: A, $0.5 \mathrm{~mm}$ ammonium chloride; $\mathrm{B}$, acetonitrile. Gradient elute procedure: $0-1.5 \mathrm{~min}, \mathrm{~B} 25-45 \%$; $1.53-2.0 \mathrm{~min}, \mathrm{~B} 45-90 \% ; 2.03-3.5 \mathrm{~min}, \mathrm{~B} 90 \% ; 3.53-$ $5.0 \mathrm{~min}, \mathrm{~B} 25 \%, 5.03-10.0 \mathrm{~min}, \mathrm{~B} 25 \%$. Column: Luna-C ${ }_{18}$, $150 \times 2.0 \mathrm{~mm}, 5 \mu \mathrm{m}$ (Phenomenex, U.S.A.), maintained at $40^{\circ} \mathrm{C}$ with flow rate of $0.2 \mathrm{ml} / \mathrm{min}$. Determination of panax notoginsenoside $\mathrm{R} 1$, ginsenoside $\mathrm{Rg} 1, \mathrm{Rd}$, $\mathrm{Re}$ and $\mathrm{Rb} 1$ was performed under this condition coupled with mass spectrometry detector.

Mass Spectrometry Detection The LC-MS2010 A series system (Shimadzu, Japan) was equipped with a binary pump, on-line vacuum degasser, autosampler, column compartment, mass spectrometry detector of electrospray interface, and LCMS Solution Version 2.04 S. The ESI ion source was set in the negative ion polarity mode for acquiring all mass spectrometry data. The selective ion monitoring (SIM) was set at $m / z 967.75$ for panax notogisenoside R1, $m / z$ 835.80 for gisenoside $\mathrm{Rg} 1, \mathrm{~m} / \mathrm{z} 981.80$ for ginsenoside $\mathrm{Rd}$ and $\mathrm{Re}, \mathrm{m} / \mathrm{z} 1143.65$ for ginsenoside $\mathrm{Rb} 1, \mathrm{~m} / \mathrm{z} 815.4$ for digoxin (internal standard). The drying gas flow, CDL temperature, block heater temperature, $\mathrm{CDL}$ voltage, probe voltage, and detector voltage were set to $1.51 / \mathrm{min}, 250{ }^{\circ} \mathrm{C}$, $200{ }^{\circ} \mathrm{C},-25 \mathrm{~V}, 4.5 \mathrm{kV}$, and $1.60 \mathrm{kV}$, respectively.

Assaying of Panax Notoginsenoside R1, Ginsenoside Rg1, Rd, Re and Rb1 in TPNS and Xuesaitong (TPNS) Injection The calibration curves of all analytes were prepared over the concentration of $10.0-160.0 \mu \mathrm{g} / \mathrm{ml}$ in methanol. TPNS and Xuesaitong (TPNS) injection were prepared at the concentration of $0.5 \mathrm{mg} / \mathrm{ml}(n=6)$. All procedures were performed on LC 2010 system (Shimadzu, Japan) with UV detector at $203 \mathrm{~nm}$ wavelength. The stabilities of QC sample at the time of 0, 2, 4, 6, 12 and $24 \mathrm{~h}$ and accuracy and repeat were investigated during our analytical process.

Pharmacokinetic Protocols of TPNS in Rats Twelve Sprague-Dawley rats (male and female, 180-220 g) were purchased from experimental animal center (China Pharmaceutical University) and used in the study after a 1-week acclimatization period. After overnight fasting, TPNS powder and Xuesaitong (TPNS) injection were administered via orally or intravenously at the dosage of 300.0 and $10.0 \mathrm{mg} / \mathrm{kg}$, respectively to each group of rats for the plasma concentration-time course study. Blood samples were col- lected in heparinized tubes at $0,0.08,0.25,0.5,0.75,1,2,3$, $4,6,8,12,24,48,72$ and $96 \mathrm{~h}$ for orally rats and $0,0.03$, $0.13,0.25,0.5,0.75,1,2,3,4,6,8,12,24,48,72$ and $96 \mathrm{~h}$ for intravenously rats respectively after dosing. Plasma was immediately separated by centrifugation at $1500 \times \boldsymbol{g}$ and stored at $-20^{\circ} \mathrm{C}$ until analysis.

Sample Preparation Extraction and cleanup of rat plasma samples were carried out by liquid-liquid extraction (LLE) according to the following procedure. To $100.0 \mu \mathrm{l}$ aliquot of rat plasma sample, $10.0 \mu \mathrm{l}$ aliquot of internal standard $(500.0 \mathrm{ng} / \mathrm{ml}$ digoxin solution) was added. The sample was briefly mixed for about $30 \mathrm{~s}$ and $n$-butanol $(1.0 \mathrm{ml})$ was added. The mixture was vortex-mixed for approximately $3.0 \mathrm{~min}$. After centrifugation at $3000 \times \boldsymbol{g}$ for $5 \mathrm{~min}, 0.8 \mathrm{ml}$ upper organic layer was removed to clean Eppendorf tube and evaporated to dryness in a Speed Vacplus Model vacuum drier. Each residue sample was reconstituted with $100.0 \mu \mathrm{l}$ methanol, vortex-mixed for $2 \mathrm{~min}$ and centrifuged at $15000 \times$ $\boldsymbol{g}$ for $10 \mathrm{~min}$, and $10.0 \mu \mathrm{l}$ of supernatant was injected onto the HPLC-MS system.

Data Analysis Content assaying of all analytes was performed according to the calibration curves on 2010 HPLC system. Determination of all analytes in rat plasma was processed on LC/ESI/MS system. The peak areas of all analytes and the internal standard were measured. The peak area ratios of analytes relative to that of the internal standard were calculated and used for construction of the standard curves. These peak area ratios were plotted against the spiked concentration of all standard analytes. Least-square linear regression was then used to determine the linearity of the curves and calculate the slope, intercept, and correlation coefficient of the line for each of the analytes. The concentration of each analytes was calculated based on the standard curve. All data is represented as mean \pm S.D.

\section{RESULTS}

Content Assaying The content of panax notoginsenoside R1, ginsenoside $\mathrm{Rg} 1, \mathrm{Rd}, \mathrm{Re}$ and $\mathrm{Rb} 1$ in TPNS and Xuesaitong (TPNS) injection was determined successfully on the condition mentioned above. All analytes in TPNS powder and Xuesaitong (TPNS) injection were stable during the analytical times of $0,2,4,6,12$ and $24 \mathrm{~h}$. The RSD\% of accuracy and repeat for all analytes was less than $11.3 \%$. Assaying results were showed in Table 1 . Content of all analytes displayed no statistic difference between TPNS powder and Xuesaitong (TPNS) injection.

Method Validation The linear regression analysis of analytes were constructed by plotting the peak area ratios of analytes to the internal standard $(y)$ versus analytes concentration spiked plasma samples $(x)$. Five sets of calibration curves were established. The mean regression equation of these curves was calculated as follows: $y=0.0028 x-0.0090$ $\left(R^{2}=0.9994\right)$ for panax notoginsenoside R1, $y=0.0025 x-$

Table 1. Assaying of Panax Notoginsenoside R1, Ginsenoside Rg1, Rd, Re and Rb1 in Total Panax Notoginsenoside (TPNS) Powder and Xuesaitong Injection $(n=6)$

\begin{tabular}{lcccc}
\hline \hline \multicolumn{1}{c}{ Preparations } & R1 & Rg1 & Re & Rb1 \\
\hline TPNS powder & $7.38 \pm 0.25$ & $26.34 \pm 0.48$ & $4.28 \pm 0.09$ & $34.66 \pm 0.55$ \\
Xuesaitong injection & $7.13 \pm 0.17$ & $25.49 \pm 0.26$ & $4.15 \pm 0.11$ & $33.78 \pm 0.35$ \\
\hline
\end{tabular}


$0.0092\left(R^{2}=0.9991\right)$ for ginsenoside $\operatorname{Rg} 1, y=0.001 x-0.0120$ $\left(R^{2}=0.9988\right)$ for ginsenoside Rd, $y=0.0011 x-0.0017\left(R^{2}=\right.$ $0.9991)$ for ginsenoside Re, $y=0.0025 x-0.0048 \quad\left(R^{2}=\right.$
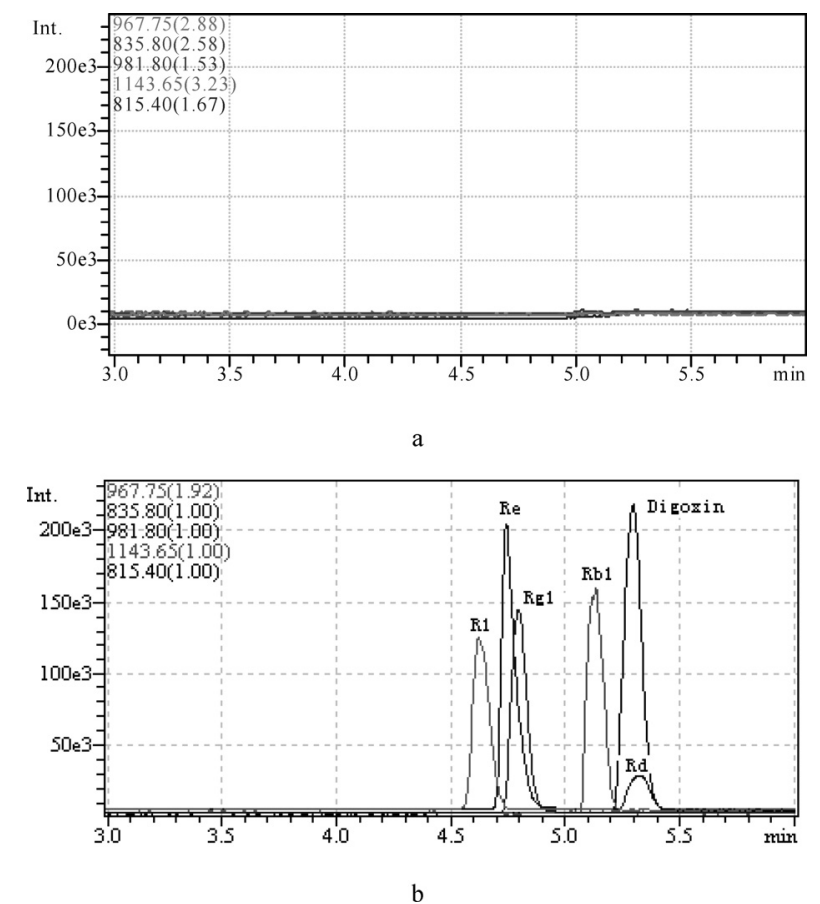

Fig. 2. Typical SIM Chromatograms of Blank Rat Plasma (a) and Panax Notoginsenoside R1, Ginsenoside Rg1, Rd, Re, Rb1 and Internal Standard Digoxin (b)
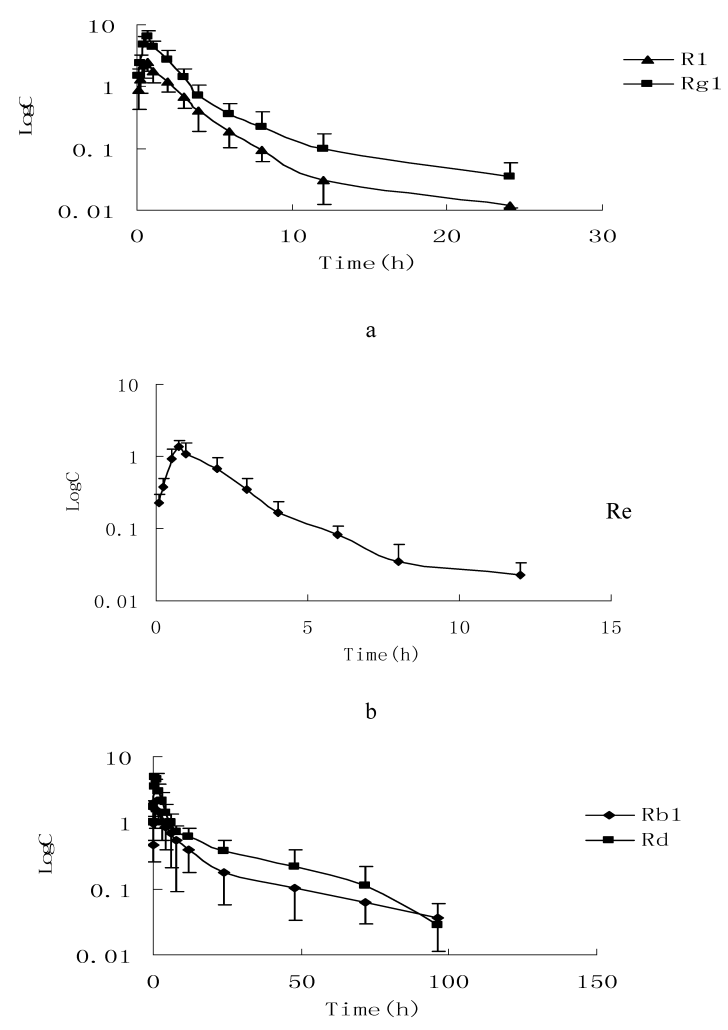

$\mathrm{c}$

Fig. 3. Mean Plasma Concentration-Time Profiles of Panax Notoginsenoside R1 and Ginsenoside Rg1 (a), Re (b), Rd and Rb1 (c) after Oral Administration Dosage of $300 \mathrm{mg} / \mathrm{kg}$ TPNS in Rats $(n=6)$
0.9990) for ginsenoside $\mathrm{Rb} 1$, which showed good linear relationships between the peak area and the concentration. The low limit of quantification (LLOQ) of R1, Rg1, Rd, Re and $\mathrm{Rb} 1$ were of $3.03,4.00,4.00,4.00$ and $2.77 \mathrm{ng} / \mathrm{ml}$ in rat plasma. The recoveries of all analytes were of $67.5-94.2 \%$.

Intra- and inter-day assay precision (CV\%) of panax notoginsenoside $\mathrm{R} 1$, ginsenoside $\mathrm{Rg} 1, \mathrm{Rd}, \mathrm{Re}$ and $\mathrm{Rb} 1$ were at the range of $1.82-11.46 \%$ over the concentration of 12.11 , 96.88 and $387.5 \mathrm{ng} / \mathrm{ml}$ for panax notoginsenoside R1, 16.02, 128.13 and $512.52 \mathrm{ng} / \mathrm{ml}$ for ginsenoside $\mathrm{Rg} 1, \mathrm{Rd}$ and $\mathrm{Re}$, $11.09,88.75$ and $355.0 \mathrm{ng} / \mathrm{ml}$ for ginsenoside $\mathrm{Rb} 1$.

The mean accuracy from the spiked concentration was from 97.52 to $102.54 \%$ for panax notoginsenoside R1, 97.62 to $100.54 \%$ for ginsenoside $\operatorname{Rg} 1,96.88$ to $100.17 \%$ for ginsenoside Rd, 97.85 to $101.14 \%$ for ginsenoside Re, 97.28 to $100.81 \%$ for ginsenoside $\mathrm{Rb} 1$ at the concentration of QC sample under the condition of $12 \mathrm{~h}$ in ambient temperature, $4{ }^{\circ} \mathrm{C} 12 \mathrm{~h}$ in sample pool, $-20{ }^{\circ} \mathrm{C}$ for four weeks and $-20^{\circ} \mathrm{C}$ for three thaw-freeze cycles.

Chromatograms and Pharmacokinetic Parameters Typical chromatograms of all analytes and digoxin (IS) were obtained and shown in Fig. 2. The mean plasma concentration-time profiles of panax notoginsenoside R1, ginsenoside $\mathrm{Rg} 1, \mathrm{Rd}, \mathrm{Re}$ and $\mathrm{Rb} 1$ after oral administration of TPNS pow$\operatorname{der}(300 \mathrm{mg} / \mathrm{kg})$ or intravenous administration of Xuesaitong (TPNS) injection $(10 \mathrm{mg} / \mathrm{kg})$ were obtained in Figs. 3 and 4 , respectively. Pharmacokinetic parameters were estimated using the DAS pharmacokinetic software (1.0 version). Maximum concentration $\left(C_{\max }\right)$ and time to maximum concentra-
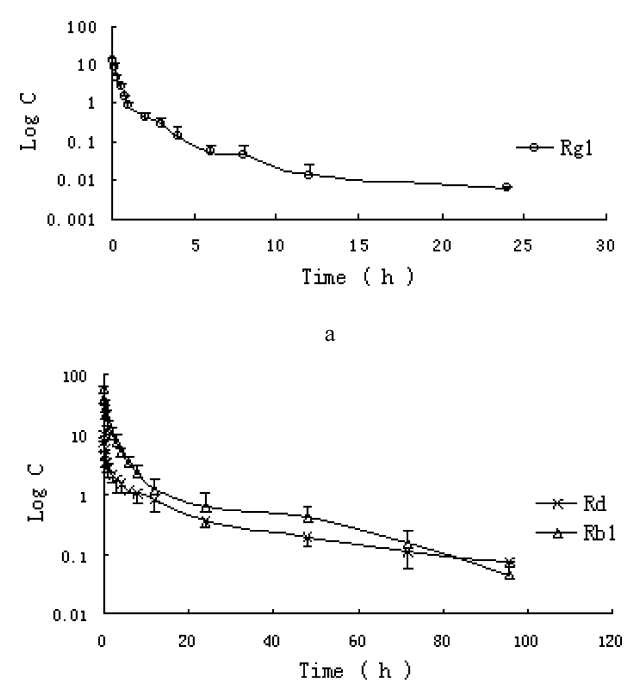

b

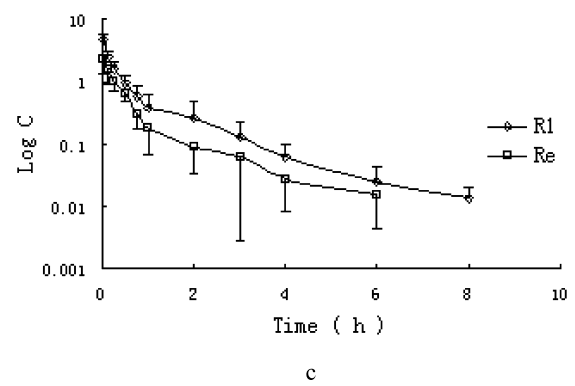

Fig. 4. Mean Plasma Concentration-Time Profiles of Ginsenoside Rg1 (a), Rd and Rb1 (b), Panax Notoginsenoside R1 and Re (c) after Intravenous Administration Dosage of $10 \mathrm{mg} / \mathrm{kg}$ Xuesaitong Injection in Rats $(n=6)$ 
Table 2. The Main Pharmacokinetic Parameters of Panax Notoginsenoside R1, Ginsenosido Rg1, Rd, Re and Rb1 after Oral Administration of 300 mg/kg Dosage of Total Panax Notoginsenoside (TPNS) in Rat Plasma $(n=6)$

\begin{tabular}{|c|c|c|c|c|c|}
\hline Parameters & $\mathrm{R} 1$ & $\operatorname{Rg} 1$ & $\mathrm{Rd}$ & $\operatorname{Re}$ & $\mathrm{Rb} 1$ \\
\hline$T_{\max }(\mathrm{h})$ & $0.71 \pm 0.19$ & $0.75 \pm 0.00$ & $0.88 \pm 0.14$ & $0.79 \pm 0.10$ & $0.83 \pm 0.13$ \\
\hline$C_{\max }(\mathrm{mg} / \mathrm{l})$ & $2.94 \pm 0.75$ & $6.42 \pm 1.74$ & $2.36 \pm 0.70$ & $1.51 \pm 0.28$ & $5.08 \pm 0.66$ \\
\hline$T_{1 / 2 \beta}(\mathrm{h})$ & $1.11 \pm 0.51$ & $5.01 \pm 2.09$ & $18.15 \pm 9.74$ & $1.01 \pm 1.35$ & $20.15 \pm 6.27$ \\
\hline$A U C_{(0-t)}(\mathrm{mg} / \mathrm{l} \cdot \mathrm{h})$ & $5.93 \pm 1.52$ & $13.89 \pm 2.54$ & $20.68 \pm 9.98$ & $3.01 \pm 1.03$ & $36.64 \pm 12.16$ \\
\hline$A U C_{(0-\infty)}(\mathrm{mg} / \mathrm{l} \cdot \mathrm{h})$ & $6.02 \pm 1.52$ & $14.10 \pm 2.52$ & $22.46 \pm 10.68$ & $3.07 \pm 1.03$ & $37.66 \pm 12.67$ \\
\hline$M R T_{(0-t)}(\mathrm{h})$ & $2.34 \pm 0.24$ & $3.16 \pm 1.05$ & $19.09 \pm 6.06$ & $2.44 \pm 0.48$ & $19.09 \pm 5.97$ \\
\hline$M R T_{(0-\infty)}(\mathrm{h})$ & $2.55 \pm 0.22$ & $3.60 \pm 1.51$ & $27.27 \pm 10.94$ & $2.73 \pm 0.55$ & $21.94 \pm 7.32$ \\
\hline
\end{tabular}

Table 3. The Main Pharmacokinetic Parameters of Panax Notoginsenoside R1, Ginsenoside Rg1, Rd, Re and Rb1 after Intravenous Administration of $10 \mathrm{mg} / \mathrm{kg}$ Dosage of Xuesaitong (TPNS) Injection in Rat Plasma $(n=6)$

\begin{tabular}{|c|c|c|c|c|c|}
\hline Parameters & $\mathrm{R} 1$ & $\operatorname{Rg} 1$ & $\mathrm{Rd}$ & $\mathrm{Re}$ & $\mathrm{Rb} 1$ \\
\hline$V_{\mathrm{d}}(1 / \mathrm{kg})$ & $12.91 \pm 5.90$ & $15.60 \pm 12.06$ & $9.65 \pm 2.59$ & $27.65 \pm 17.18$ & $6.20 \pm 4.07$ \\
\hline$T_{1 / 2 \beta}(\mathrm{h})$ & $1.67 \pm 0.30$ & $4.03 \pm 2.75$ & $19.24 \pm 3.05$ & $0.72 \pm 0.45$ & $22.16 \pm 13.64$ \\
\hline$C L(1 / \mathrm{h} / \mathrm{kg})$ & $5.22 \pm 1.73$ & $1.91 \pm 0.33$ & $0.46 \pm 0.35$ & $10.72 \pm 3.47$ & $0.12 \pm 0.05$ \\
\hline$A U C_{(0-t)}(\mathrm{mg} / \mathrm{l} \cdot \mathrm{h})$ & $2.13 \pm 0.80$ & $7.71 \pm 4.12$ & $30.91 \pm 9.24$ & $1.43 \pm 0.44$ & $105.27 \pm 36.28$ \\
\hline$A U C_{(0-\infty)}(\mathrm{mg} / \mathrm{l} \cdot \mathrm{h})$ & $2.16 \pm 0.79$ & $7.76 \pm 4.11$ & $31.72 \pm 9.48$ & $1.45 \pm 0.45$ & $106.44 \pm 36.71$ \\
\hline
\end{tabular}

tion $\left(T_{\max }\right)$ were the experimentally observed values. The area under the plasma concentration-time curve (AUC) was calculated using the trapezoidal rule. $A U C_{t}$ was calculated from time zero to real time and $A U C_{\infty}$ from time zero to infinity. Apparent elimination half-life $\left(t_{1 / 2}\right)$ was calculated as $T_{1 / 2}=0.693 / k_{\mathrm{e}}$ where $k_{\mathrm{e}}$ means the terminal elimination rate constant. The absolute bioavailability $(F \%)$ was estimated from the dose-normalized ratios of $\left[A U C_{\infty}\right]_{\text {p.o. }}$ to $\left[A U C_{\infty}\right]_{\text {i.v. }}$. The estimated pharmacokinetic parameters of all analytes after oral or intravenous administration were shown in Tables 2 and 3 respectively. The absolute bioavailability of panax notoginsenoside $\mathrm{R} 1$, ginsenside $\mathrm{Rg} 1, \mathrm{Rd}, \mathrm{Re}$ and $\mathrm{Rb} 1$ were of $9.29 \%, 6.06 \%, 2.36 \%, 7.06 \%$ and $1.18 \%$, respectively.

\section{DISCUSSIONS}

After preliminary testing, LC/ESI/MS method was developed and validated for the simultaneous determination of panax notoginsenoside $\mathrm{R} 1$, ginsenoside $\mathrm{Rg} 1$, $\mathrm{Rd}, \mathrm{Re}$ and $\mathrm{Rb} 1$ in rat plasma instead of TLC, ${ }^{11)} \mathrm{HPLC}^{10,12-14)}$ and LC/MS/ $\mathrm{MS}^{15,16)}$ for their shortcoming of poor sensitivity, specificity, selectivity and expensive cost. There was no any endogenous matrix interference with all analytes and all chromatographic run time were within $10.0 \mathrm{~min}$.

Many researchers focused on total panax notoginsenoside (TPNS) because of its multiple constituents and important role in clinic during the past several decades. However, no any further more progress obtained on the pharmacokinetic evaluation of TPNS because of its multiple constituents. It was very difficult for us to choose suitable constituents to evaluate the pharmacokinetics profiles of TPNS because of its multiple constituents and the limit of analytical technology. Rg1 and Rb1 were often considered for high content in TPNS. Odani research group reported the ADME profiles of ginsenoside $\mathrm{Rg} 1$ and $\mathrm{Rb} 1$ isolated from panax ginseng in rats in 1980s. ${ }^{11,17)}$ Pharmacokinetic profiles of TPNS reported recent years according to the pharmacokinetic evaluation of ginsenoside $\mathrm{Rg} 1$ and $\mathrm{Rb}^{9-12)}$ in rat. However, it was a kind of jug-handled research only focus on $\mathrm{Rg} 1$ and $\mathrm{Rb} 1$. TPNS is a kind of typical multiple constituent TCM widely used in oriental countries and it is necessary for the systematic pharmacokinetic evaluation of TPNS. According to the content assaying of TPNS in this study (Table 1) we know that panax notoginenoside $\mathrm{R} 1$, ginsenoside $\mathrm{Rg} 1, \mathrm{Rd}, \mathrm{Re}$ and $\mathrm{Rb} 1$ are the main constituents of TPNS. The total percentage of these constituents is more than $78.53 \%$ in TPNS. It is reasonable and necessary for the pharmacokinetic study of panax notoginsenoside $\mathrm{R} 1$, ginsenoside $\mathrm{Rg} 1, \mathrm{Rd}, \mathrm{Re}$ and $\mathrm{Rb} 1$ when the pharmacokinetic profile of TPNS being evaluated in rats. In this study, LC/ESI/MS method was successfully employed for the pharmacokinetic evaluation of TPNS in rats.

Main pharmacokinetic parameters of these constituents mentioned above were obtained by DAS pharmacokinetic software recommendation from Chinese Pharmacological Association. From the results we know that after oral administration of TPNS powder in rats, panax notoginsenoside R1, ginsenoside $\mathrm{Rg} 1, \mathrm{Rd}, \mathrm{Re}$ and $\mathrm{Rb} 1$ reached peak concentration in plasma rapidly within about $0.75 \mathrm{~h}$ which hint their absorption were quickly. Maximum concentration of R1, $\mathrm{Rg} 1, \mathrm{Rd}, \mathrm{Re}$ and $\mathrm{Rb} 1$ in rat plasma were between 1.51 to $6.42 \mu \mathrm{g} / \mathrm{ml}$ means their poor absorption. $T_{1 / 2}$ was changed from 1.01 to $20.15 \mathrm{~h}$ for 5 analytes in rat plasma. After intravenous administration of Xuesaitong (TPNS) injection in rats, most of constituents were cleared quickly except ginsenoside $\mathrm{Rd}$ and $\mathrm{Rb} 1 . T_{1 / 2}$ was changed from 0.72 to $22.16 \mathrm{~h}$, $V_{\mathrm{d}}$ from 6.20 to $27.65 \mathrm{l} / \mathrm{kg}$ and $C L$ from 0.12 to $10.72 \mathrm{l} / \mathrm{h} / \mathrm{kg}$ for all analytes. Absolute bioavailability study suggested that all ginsenosides in TPNS were poorly absorbed by rats. The reasons maybe included that these saponins were destroyed in gastrointestinal tract, metabolized by intestinal microflora $^{18)}$ and excreted from bile or urine. The variability of $T_{1 / 2}, V_{\mathrm{d}}, C L$ and $A U C$ for all analytes maybe related to the binding ratio of plasma protein (ginsenoside $\mathrm{Rg} 1$ was of low ratio whereas $\mathrm{Rb} 1$ was high ${ }^{17)}$ ). 


\section{CONCLUSION}

A rapid, sensitive, selective and specific LC/ESI/MS method was developed and validated for the simultaneous determination of panax notoginsenoside R1, ginsenoside $\mathrm{Rg} 1, \mathrm{Rd}, \mathrm{Re}$ and $\mathrm{Rb} 1$ in rat plasma and successfully employed for the pharmacokinetic and absolute bioavailability study of TPNS and Xuesaitong injection. It maybe offer us a kind of platform for the pharmacokinetic evaluation of multiple constituent traditional Chinese medicine (TCM). A hypothesis was originated from these results: the multiple target effect mechanism of multiple constituent TCM probably due to the pharmacokinetic profile diversities of these constituents.

Acknowledgements The project was supported by the National High Technology Foundation of China ("863" Projects) for preclinical pharmacokinetic studies (No: 2005AA2Z3C70), National Natural Science Foundation of China (No: 30572228 and key project No: 30630076), Jiangsu Natural Science Foundation of China (No: BK2004111).

\section{REFERENCES}

1) Vongsangnak W., Gua J., Chauvatcharin S., Zhong J. J., J. Biochem. Eng., 18, 115-120 (2004).
2) Ma W. G., Malterud K. E., Lu S. L., Ducrey B., Tahara S., Mizutani M., Phytochemistry, 54, 1133-1139 (1999).

3) Liu S. J., Zhou S. W., Acta Pharmacol. Sin., 21, 257-260 (2000).

4) Zou K., Zhu S., Tohda C., Cai S. Q., Komatsu K., J. Nat. Prod., 65, 346-351 (2002).

5) Ma L. Y., Xiao P. G., Phytother. Res., 12, 138-140 (1998)

6) Attele A. S., Wu J. A., Yuan Ch. S., Biochem. Pharmacol., 58, 16851693 (1999).

7) Lai C. M., Li S. P., Yu H., Wan J. B., Kan K. W., Wang Y. T., J. Pharm. Biomed. Anal., 40, 669-678 (2006).

8) Han M., Sha X. Y., Wu Y. J., Fang X. L., Planta Med., 72, 398-404 (2006).

9) Xu Q. F., Fang X. L., Chen D. F., J. Ethnopharmacol., 84, 187-192 (2003).

10) Li L., Sheng Y. X., Zhang J. L., Wang Ch. Sh., Guo D. A., Biomed. Chromatogr., 18, 849-856 (2004).

11) Odani T., Tanizawa H., Takino Y., Chem. Pharm. Bull., 31, 292-298 (1983).

12) Li L., Zhang J. L., Sheng Y. X., Guo D. A., Wang Q., Guo H. Z., J. Pharm. Biomed. Anal., 38, 45-51 (2005).

13) Li W. K., Fitzloff J. F., J. Pharm. Pharmacol., 53, 1637-1643 (2001).

14) Shangguan D. H., Han H. W., Zhao R., Zhao Y. X., Xiong S. X., Liu G. Q., J. Chromatogr. A, 910, 367-372 (2001).

15) Lee P. S., Song T. W., Sung J. H., Moon D. C., Song S., Chung Y. B., Planta Med., 72, 204-210 (2006).

16) Li K., Chen X. Y., Xu J. H., Li X., Zhong D. F., Rapid Commun. Mass Spectrom., 19, 813-817 (2005).

17) Odani T., Tanizawa H., Takino Y., Chem. Pharm. Bull., 31, 10591066 (1983).

18) Bae E. A., Shin J. E., Kim D. H., Biol. Pharm. Bull., 28, 1903-1908 (2005). 